\title{
Computer simulation of copolymer phase behavior
}

\author{
Andrew J. Schultz, Carol K. Hall, ${ }^{\text {a) }}$ and Jan Genzer \\ Department of Chemical Engineering, North Carolina State University, Raleigh, North Carolina 72695
}

(Received 11 February 2002; accepted 17 September 2002)

\begin{abstract}
Discontinuous molecular dynamics simulation is used to study the phase behavior of diblock copolymers modeled as chains of tangent hard spheres with square shoulder repulsions between unlike species as a function of chain length, volume fraction and interaction strength $(\chi)$. The location of the order-disorder transition for a symmetric copolymer is close to the predictions of Fredrickson and Helfand. Our simulation results for packing fractions of 0.35, 0.40, and 0.45 and chain lengths 10 and 20 are summarized in phase diagrams which display disordered, lamellae, perforated lamellae (PL), cylindrical, and BCC spherical (S) phases in the $\chi N$ versus $f$ plane. These phase diagrams are consistent with phase diagrams from other simulation studies. Contrary to theoretical predictions we observe the PL phase near regions of predicted gyroid stability, and the $\mathrm{S}$ phase only in the systems with high packing fraction and long chain length. These discrepancies may be due to the short chain lengths considered, as they are less evident in the 20-bead chains than the 10-bead chains. We examine the structural spacing of the microphases and the variation of that spacing with $\chi N$. We also examine the internal energy and entropy and their variation with $\chi N$. Our results are consistent with self-consistent field theory results for the strong segregation limit.

(C) 2002 American Institute of Physics. [DOI: 10.1063/1.1519839]
\end{abstract}

\section{INTRODUCTION}

Interest in block copolymers has grown considerably in recent years because their unique microphase behavior is expected to play a prominent role in future technologies such as high-density magnetic storage materials, molecular semiconductors, optical coatings and catalysts. Unlike blends of homopolymers whose phase separation occurs on a macroscopic level with separate domains formed for each polymer, block copolymers' phase separation is microscopic because the two components are chemically linked. The resulting microphases exhibit classical morphologies, such as lamellae, cylinders, and spheres, and complex morphologies, such as perforated lamellae and gyroid. Because of the molecularlevel separation between components, materials can be developed which order on a much smaller scale than that found in traditional materials.

The phase behavior of block copolymers has received considerable theoretical attention from the polymer community, with studies examining the behavior both above and below the order-disorder transition (ODT), the point at which an ordered structure forms from a disordered state. ${ }^{1}$ Theories have been developed to predict the ODT and the order-order transitions (OOT), the spacing between structural units such as lamellar sheets or cylinders, ${ }^{2-10}$ and the thickness of the interface between domains ${ }^{9,10}$ as a function of the copolymer length $(N)$, the volume fraction of each component $(f)$ along the chain, and the strength of interaction $(\chi)$. The major contributions to the free energy in these theories are the interfacial energy and the entropy of chain stretching. Liebler used mean-field theory to predict the phase diagram for a block copolymer system. ${ }^{2}$ Fredrickson

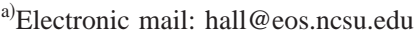

and Helfand and later Barrat and Fredrickson reformulated Liebler's mean-field theory to include fluctuations, which effectively raised the value of $(\chi N)_{\mathrm{ODT}}$ and brought predictions of periodic spacing into agreement with experiment for the weak segregation regime (WSR), where $\chi N$ is small. ${ }^{3,4}$ Semenov developed an alternative mean-field theory which is valid in the strong segregation regime (SSR), where $\chi N$ is large, and used it to predict phase boundaries and periodic spacing between structural units. ${ }^{9}$ Banaszak and Whitmore used self-consistent field theory (SCFT), which allows the predicition of properties in the SSR and the intermediate segregation regime (ISR), which is between the WSR and SSR, to construct a phase diagram for block copolymers in solvents. ${ }^{11}$ They also examined the periodic spacing as a function of interaction strength, $f, N$, and copolymer volume fraction, $\phi_{c}$. Matsen and Schick formulated a new version of SCFT without the unit cell approximation, and were able to predict the stability of the experimentally observed gyroid phase. ${ }^{12}$ Later, Matsen and Bates used this new formulation to examine thermodynamic properties such as the internal energy and entropy, and structural properties such as the interfacial width, interfacial area, periodic spacing and mean curvature. ${ }^{7}$

Block copolymer phase separation has also been studied via computer simulation which is able, in principle, to provide more microscopic-level information than experiment. The most popular method for simulating block copolymers has been lattice Monte Carlo, chosen because of its speed relative to other methods and its ability to accommodate very large structures. A disadvantage of lattice Monte Carlo is that the lattice cannot stretch to accommodate a particular structure. Larson used lattice Monte Carlo to study symmetric block copolymers of lengths ranging from 6 to 192 in selective solvents. He examined the location of the order-disorder 
transition, concentration profiles, and periodic spacing of lamellar structures at the ODT. ${ }^{13}$ Besold and co-workers studied a system of diblock copolymers of length 30 using lattice Monte Carlo. ${ }^{14}$ They found lamellar and cylindrical structures, and examined the ODT for symmetric copolymers. Hoffman and co-workers studied symmetric and asymmetric chains of length 24 , and found disordered, lamellar, and cylindrical structures. ${ }^{15,16}$ They monitored static properties, such as the structure factor and the radius of gyration, and also dynamic properties such as diffusion and relaxation.

Off-lattice molecular dynamics simulations have also been performed; these have the advantage that they can accommodate most structures and the disadvantage that they are relatively slow. Murat and co-workers used molecular dynamics to study symmetric block copolymers modeled as chains of truncated Lennard-Jones spheres. ${ }^{17}$ They monitored the periodic spacing, the radius of gyration, the end-to-end distance, and the diffusion constant, and examined the effect of varying the chain length and strength of interaction. Groot and Madden used dissipative particle dynamics to study the phases formed at different volume fractions, and found disordered, lamellar, perforated lamellar, cylindrical, and bcc spherical phases. ${ }^{18}$

In this paper, we describe the results of discontinuous molecular dynamics (DMD) simulations of diblock copolymer systems. Our block copolymer is modeled as a hardsphere chain with an additional square shoulder repulsion between unlike segments. We have conducted simulations of chains of lengths 10 and 20 at packing fractions $\eta=0.35$, 0.40 and 0.45 and volume fractions $f=0.1,0.2,0.3,0.4$, and 0.5 . In order to accommodate the periodic spacing of different structures, we have used an algorithm that allows the box lengths to be adjusted to accommodate different structures or structural spacings. We have also investigated the stability of the resulting structures using Escobedo and de Pablo's EVALENCH algorithm to calculate the free energy. ${ }^{19}$

Based on our simulations, we have constructed phase diagrams for the systems at different chain lengths and packing fractions. We find disordered, lamellar, cylindrical, perforated lamellar, and bcc spherical structures. Examples of these structures, except bcc spheres, are shown in Fig. 1. In constructing the phase diagram, we have encountered metastable structures. In these cases, we have identified the most stable structure by calculating the free energy. The resulting phase diagrams are consistent with other simulation studies, but differ qualitatively from mean-field theory predictions.

We have examined the periodic spacing between repeat units of the structures. We report the variation of the periodic spacing with interaction strength and chain length for the lamellar morphology. The periodic spacing behavior is similar to theoretical predictions for the SSR. Quantitative agreement improves with increasing chain length.

Finally, we have calculated the internal energy and entropy of the lamellar phase. These quantities are qualitatively consistent with the results of SCFT, with minor differences. Agreement between our internal energy results and SCFT improves with chain length, but the agreement between our entropy results and SCFT does not.

The remainder of this paper is organized into the follow-

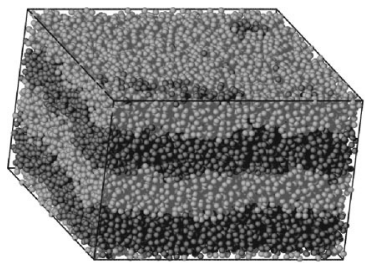

Lamellae at $\mathrm{f}=0.5$ and $\mathrm{cN}=48.6$

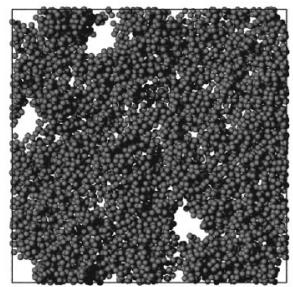

Perforated lamellae at $\mathrm{f}=\mathbf{0 . 4}$ and $\mathrm{cN}=32.4$ Only the majority component is shown

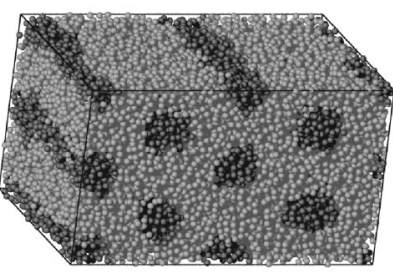

Cylinders at $\mathrm{f}=0.2$ and $\mathrm{cN}=97.3$



Disordered phase at $\mathrm{f}=0.5$ and $\mathrm{cN}=24.3$
FIG. 1. Simulation structures for $N=20$.

ing sections: Section II describes the copolymer model and the molecular simulation techniques that we have used. Section III presents the phase diagrams resulting from our simulations. Section IV describes the properties of the symmetric copolymer systems, including the periodic spacing, the internal energy and entropy. In Sec. V, we summarize our results and discuss future avenues of research.

\section{COPOLYMER MODEL AND SIMULATION TECHNIQUE}

\section{A. Copolymer model}

The copolymer is modeled as a flexible chain of spheres, with component $\mathrm{A}$ spheres on one end and component $\mathrm{B}$ spheres on the other. All beads interact with a hard sphere potential of diameter, $\sigma$. Additionally, components A and B repel each other with a square-shoulder potential, $\epsilon_{\mathrm{AB}}$, which extends to an intermolecular separation $2 \sigma$. The resulting potentials are

$$
\begin{aligned}
& U_{\mathrm{AA}}(r)=U_{\mathrm{BB}}(r)= \begin{cases}\infty & \text { if } r<\sigma \\
0 & \text { if } r>\sigma,\end{cases} \\
& U_{\mathrm{AB}}(r)= \begin{cases}\infty & \text { if } r<\sigma \\
\epsilon & \text { if } \sigma<r<2 \sigma \\
0 & \text { if } r>2 \sigma .\end{cases}
\end{aligned}
$$

We have chosen to have the two components repel each other rather than to have an attractive potential between like components because this significantly increases computational efficiency. To see this, consider the case in which the system is ordered, and hence contains domains of A and B. Since we only have interactions (repulsive shoulder) between unlike monomers, interactions within each domain are purely hard-sphere interactions and proceed quickly. The only particles to experience the more computationally expensive square-shoulder potential interactions are those at the interface. If we were to have an attractive potential between like components, the square-well interactions would exist 
throughout each domain, and even into the interface, where at least half of the interactions would have a square-well component. From a physical perspective, the two sets of potentials are roughly equivalent because $\chi$, the parameter which determines the phase behavior of the copolymers, is the same for both. In addition to these advantages, hard-coreonly potentials between like components offer the advantage that the chains do not experience thermal expansion.

\section{B. DMD simulation algorithm}

In a discontinuous molecular dynamics (DMD) simulation, particles experience collisions when they hit a discontinuity in the potential. Between collisions, particles experience linear trajectories, making the simulation technique faster than traditional molecular dynamics simulations which generally require a small integration time step. The postcollision velocities can be found by solving the collision dynamics equations. In order to treat chains of beads effectively, Rapaport created bonds between beads by restricting the distance between adjacent beads to lie between $\sigma$ and $\sigma(1+\delta) .{ }^{20}$ Bellemans later extended this model so that the distance between adjacent beads can lie between $\sigma(1$ $-\delta / 2)$ and $\sigma(1+\delta / 2)$, making the average bond length $\sigma{ }^{21}$

We have developed a DMD code suitable for simulating copolymers and any mixture of monomers, polymers, and copolymers. This code was adapted from code originally developed by Smith and co-workers. ${ }^{22}$ It includes a search algorithm that allows the system to adjust its box length in three dimensions in order to accommodate ordered structures that might form. It is also optimized to allow for the efficient simulation of large multicomponent systems.

\section{Box length search algorithm}

Our DMD code includes an algorithm that adjusts the box dimensions to accommodate different structures or structural spacings that might try to form over the course of the simulation. Ordered structures have periodic spacing in at least one dimension. Because the periodic spacing has a particular value which minimizes the free energy of the system, the box length in the direction, $k$, of the periodic spacing must be a multiple of that periodic spacing. If the box lengths are incorrect, they can change not only the periodic spacing, but by increasing the free energy, can give erroneous results as to which phase has the lowest free energy (e.g., cylinders instead of lamellae). Therefore, it is important to find the correct box lengths for the system. This is accomplished by comparing the pressure in the three orthogonal directions. If the box length in the $k$ direction is not long enough, then the structure will be compressed in that direction, and the pressure in that direction will be higher than it would be if the box length were correct. If there is another dimension, $l$, in the system without periodic spacing, then the system will not tend to become compressed or stretched in that direction, and the pressure in that direction will be independent of the box length in that direction. So, if the pressures in directions $k$ and $l$ are different, this tells us that the system is either stretched or compressed, and that the box lengths should be changed in order to minimize the free energy of the system.
We have developed an algorithm to find the box lengths appropriate to a specific packing fraction. The algorithm searches for box lengths which maintain the packing fraction at a specific value, but equalize the pressure in the three directions. Unfortunately, some structures (spheres, HPL, and gyroid) have periodic spacing in all three directions, which means that the pressure algorithm, by itself, is insufficient to find the correct box lengths for these phases, because changing all three box lengths independently would require changing the packing fraction. Instead, in such cases we use a box size that is sufficiently large that the system can reorient to vary the effective length of the unit cell. A large box size also allows the system to adjust the number of repeat units per box dimension.

The first step in the algorithm is to run the simulation at constant volume and calculate the reduced pressure in each direction using the virial theorem

$$
P_{k}^{*}=\frac{P_{k} \sigma^{3}}{k_{B} T}=\frac{N_{b} \sigma^{3}}{V}-\frac{\sigma^{3} m \Sigma_{\mathrm{coll}} r_{i j k} \Delta v_{j k}}{V k_{B} T t},
$$

where $k$ is the direction ( $k=x, y$, or $z$ ), $k_{B}$ is Boltzmann's constant, $T$ is the temperature, $N_{b}$ is the number of beads, $V$ is the volume, $m$ is the mass of each segment, $r_{i j k}$ is the $k$ component of the distance between colliding particles $i$ and $j$, $\Delta v_{j k}=-\Delta v_{i k}$ is the $k$ component of the velocity change for the particle $j$, and $t$ is the elapsed simulation time.

The second step is to compare the pressure in one direction, $k$, with the pressure in the other two directions. If the pressure in one of these two directions, say $l$, differs from the pressure in the $k$ direction, $P_{k}^{*}$, by more than $\delta P_{\text {tol }}^{*}$, then the box length in the $l$ direction is changed accordingly. At the same time, the box length in the $k$ direction is changed to maintain constant volume. The coordinates of all beads in the system are scaled up or down with the box lengths. The size of the box length change is restricted due to the discontinuous nature of the potential and the bonding between segments. Excluded volume limits box compression, while the maximum bond length limits box expansion. The box length adjustments are performed periodically until the new box lengths have been achieved. These two steps are repeated periodically throughout the simulation until each box length fluctuates around a certain value.

The sensitivity of the box length search algorithm can be controlled by changing the algorithm parameters. By increasing the frequency of pressure checking, the system is quicker to respond to differences in pressure. By decreasing the frequency of pressure checking, the system is slower to respond, but the volume fluctuates less due to better averaging in the virial sum. A similar effect can be achieved by adjusting $\delta P_{\text {tol }}^{*}$. The two parameters (frequency and tolerance) should generally be adjusted together, so that a higher tolerance is used when the frequency is higher.

One of the advantages of the box length search algorithm is that box length fluctuations are suppressed (or minimized). This is important because large fluctuations would slow the search and complicate determination of the end point. Fluctuations are suppressed by ignoring pressure fluctuations with amplitudes less than $\delta P_{\text {tol }}^{*}$ or frequencies greater than the frequency of pressure checking. Because the 
algorithm is not "distracted" by these fluctuations, it can direct the box lengths to their optimal values more efficiently.

Other possible methods that could be used to find the appropriate box length are the hybrid DMD-MC constant pressure algorithm developed by Wilson and Allen, ${ }^{23}$ and used by Kenkare and co-workers, ${ }^{24}$ or the piston-like constant pressure algorithm of Parrinello, ${ }^{25}$ which was used by Murat and co-workers. ${ }^{17}$ The DMD-MC hybrid method involves making random box length change attempts periodically throughout the simulation. These moves are accepted or rejected based on the change in potential energy that would result if the move were accepted. Parrinello's algorithm treats the simulation box like the chamber of a piston with constant external pressure. If the pressure within the box is different than the external pressure, an imaginary force is applied to the piston. This force accelerates the piston according to Newton's second law, which can be integrated in time to regulate box length changes. Although these methods are well suited to conducting constant pressure simulations, they are not as efficient as our algorithm if the only purpose is to find the appropriate box lengths. In fact, neither of these other algorithms maintains the total volume of the system at a constant value during the simulation.

Finally, we should point out that the box length search algorithm described here is neither ergodic (like the hybrid DMD-MC algorithm) nor dynamic (like the piston algorithm). An ergodic algorithm must be reversible: At equilibrium, the probability of the system going from state A to state B must be equal to the probability of going from state B to A. Our algorithm is designed to take the system from one state to another and suppress fluctuations. If it goes from state A to B, it is unlikely to go back to A. A dynamic algorithm must attempt to mimic the dynamics of a real system by responding to the forces according to the laws of physics. Our algorithm responds to forces in a heuristic way that is different than the way that the laws of physics require. However, since the box length search algorithm is not used to calculate any properties and is only used to adjust the shape of the simulation box, the lack of ergodicity and dynamics does not adversely affect our results.

\section{Relating simulation parameters to commonly accepted polymer parameters}

It is useful to be able to relate the parameters in our model to the parameters commonly used in polymer theory and experiment: the statistical segment length, $a$, the effective chain length for fluctuations, $\bar{N}$, and the interaction strength, $\chi$. This allows us to compare our results with theoretical predictions and experimental results.

The statistical segment length, $a$, can be calculated in terms of the simulation values for $R_{g}^{\text {athermal }}$, the athermal radius of gyration in the melt, using the equation

$$
\left(R_{g}^{\text {athermal }}\right)^{2}=\frac{a^{2}}{6} N .
$$

The effective copolymer length for fluctuations, $\bar{N}$, is chosen to be the Ginsburg parameter. The Ginsburg param- eter is defined in terms of simulation values for $R_{g}^{\text {athermal }}$ and $\rho_{c}$, the copolymer number density, using the following equation: ${ }^{26}$

$$
\bar{N} \equiv 6^{3}\left(\left(R_{g}^{\text {athermal }}\right)^{3} \rho_{c}\right)^{2} .
$$

This effective length determines the impact of fluctuations on copolymer behavior in theories by Fredrickson and Helfand, and by Barrat and Fredrickson.

The interaction strength, $\chi$, can be calculated from

$$
\chi \equiv z\left(\epsilon_{\mathrm{AB}}-\frac{1}{2}\left(\epsilon_{\mathrm{AA}}+\epsilon_{\mathrm{BB}}\right)\right) / k_{B} T,
$$

where $z$ is the number of nonbonded neighbors within the interaction distance, $k_{B}$ is Boltzmann's constant, $T$ is the temperature and $\epsilon_{i j}$ is the interaction energy between components $i$ and $j$. Since $\epsilon_{\mathrm{AA}}=\epsilon_{\mathrm{BB}}=0$ in our work, this formula reduces to $\chi=z \epsilon_{\mathrm{AB}} / k T$, with $z$, the average number of nonbonded neighbors within the interaction range, $2 \sigma$, coming from our simulation data. Although Eq. (6) is only strictly correct when the density is homogeneous (which is the case for an incompressible system), the total density in our simulations is nearly homogeneous, except at strong segregation, which we discuss in Sec. IV A. The value of $z$ is nearly proportional to density and is equivalent to $z_{\text {eff }}=\varphi z^{\prime}$ in a lattice simulation, ${ }^{13}$ where $z^{\prime}$ is the number of neighboring lattice sites and $\varphi$ is the volume fraction of occupied sites. This equation for $z_{\text {eff }}$ does not apply for off-lattice simulations, so we must calculate $z_{\text {eff }}$ directly. Consequently our $\chi$ is the equivalent of the lattice $\chi_{\text {eff }}$, defined in lattice simulations as a function of $\varphi$ and the $\mathrm{AB}$ interaction parameter, $\chi_{\mathrm{AB}}=z^{\prime} \epsilon_{\mathrm{AB}} / k T$. In other words

$$
\begin{aligned}
\chi_{\text {eff }} & \equiv \varphi \chi_{\mathrm{AB}} \\
& =\varphi z^{\prime} \epsilon_{\mathrm{AB}} / k T .
\end{aligned}
$$

We will refer to it simply as $\chi$ in the rest of the paper. We shall see that using a density-dependent $\chi$ allows us to more effectively analyze density-dependent phenomena.

Values of $z, \chi, R_{g}^{\text {athermal }}, \bar{N}$ from our simulations at different packing fractions and chain lengths can be found in Table I. We also include the range of compressibility factors, $Z$ at $f=0.5$, over the temperatures considered.

\section{Simulation methods}

We have performed simulations of copolymer chains containing 10 and 20 beads at packing fractions, $\eta$, of 0.35 , 0.40 , and 0.45 , where $\eta \equiv \pi N_{b} \sigma^{3} / 6 \mathrm{~V}, \mathrm{~V}$ is the volume, and $N_{b}$ is the number of beads in the system. The phase behavior and periodic spacing were recorded as a function of volume fraction, $f$, packing fraction, $\eta$, and reduced temperature, $T^{*} \equiv k_{B} T / \epsilon_{\mathrm{AB}}$. We started our simulations in a random configuration of 125 (200 for 20-bead chains) chains at the desired packing fraction with equal box lengths on all sides. The temperature was set to a temperature at which we expected a structure to form, and a simulation was performed using the box length search algorithm described in Sec. II B 1 . Once the algorithm converged (signaled by having each box length fluctuate about a certain value), we replicated two copies of the unit subcell in every direction yield- 
TABLE I. Values of $z, \chi, R_{g}^{\text {athermal }}, a, \bar{N}$, and $Z$ from our simulations at different packing fractions, $\eta$, and chain lengths, $N$.

\begin{tabular}{cccccccc}
\hline \hline$N$ & $\eta$ & $z$ & $\chi$ & $R_{g}^{\text {athermal }}$ & $a$ & $\bar{N}$ & $Z$ \\
\hline 10 & 0.35 & 19.54 & $19.54 / T^{*}$ & 1.5408 & 1.1935 & 12.914 & $33-43$ \\
10 & 0.40 & 22.63 & $22.63 / T^{*}$ & 1.5113 & 1.1706 & 15.020 & $46-51$ \\
10 & 0.45 & 25.82 & $25.82 / T^{*}$ & 1.4828 & 1.1429 & 16.958 & $65-72$ \\
20 & 0.35 & 19.46 & $19.46 / T^{*}$ & 2.3046 & 1.2623 & 36.150 & $59-66$ \\
20 & 0.40 & 22.56 & $22.56 / T^{*}$ & 2.2629 & 1.2394 & 42.316 & $84-93$ \\
20 & 0.45 & 25.74 & $25.74 / T^{*}$ & 2.2131 & 1.2122 & 46.863 & $119-130$ \\
\hline \hline
\end{tabular}

ing 1000 (1600 for the 20-bead chains) chains, and continued the simulation again using the box length search algorithm. At the end of this process, ordered structures were observed. For example, lamellar structures formed containing two lamellar repeat units (abab) and cylindrical systems formed containing eight cylinders. Once the larger system reached equilibrium, we stopped the box length search simulation and started an NVT simulation so that we could collect data. Average CPU time needed on our $600 \mathrm{MHz}$ Alpha 21164 included $13 \mathrm{~h}$ for the equilibration of the small systems, 2.5 days for equilibration of the large systems, and $30 \mathrm{~h}$ for the data collection for the large system at each state point.

The phase diagrams described in the next section were created by performing the simulations described above over a wide variety of temperatures and volume fractions. Temperature sweeps were conducted by starting at low temperature and then increasing the temperature. Volume fraction sweeps were conducted by starting at a volume fraction of 0.5 and then decreasing it by reassigning components to different beads.

\section{E. Calculation of free energy and entropy}

We have used the expanded ensemble technique, EVALENCH (expanded variable-length chain method), developed by Escobedo and de Pablo, to calculate the chemical potential of our copolymer molecules. A detailed description of the expanded ensemble technique can be found in Refs. 19 and 27. Here, we present a brief overview.

The EVALENCH algorithm is similar in concept to the Widom insertion method ${ }^{28}$ except that a molecule is inserted and deleted in pieces (two beads at a time) during the simulation. These insertions and deletions are accepted with transition probability dictated by the weighted Metropolis criteria

$$
P_{i \rightarrow j}=\min \left[1, \exp \left(-\Delta U_{i \rightarrow j} / k T\right) w_{i} / w_{j}\right],
$$

where $U_{i \rightarrow j}$ is the change in energy associated with going from state $i$ to state $j$ (such as from 0 beads to 2 beads), and $w_{i}$ is the weight assigned to state $i$. The weights are adjusted so that the probability, $P_{i}$, that the system is in state $i$ is approximately equal for all states. This allows the system to move more freely between the different states and improves the sampling of the algorithm.

The excess chemical potential (relative to the ideal gas) of the whole molecule is the sum of contributions to the excess chemical potentials associated with inserting the vari- ous parts of the molecule. The excess chemical potential contribution for going from state $i$ to state $i+1$ is

$$
\mu_{i \rightarrow i+1} / k T=\ln \left(\frac{w_{i} P_{i+1}}{P_{i} w_{i+1}}\right) \text {. }
$$

The excess chemical potential of the whole molecule is then

$$
\mu_{\mathrm{ex}} / k T=\ln \left(\frac{w_{0} P_{N}}{P_{0} w_{N}}\right),
$$

where states $i=0$ and $i=N$ are completely deleted and inserted molecules, respectively.

During our molecular dynamics simulation, we periodically perform EVALENCH bead insertions and deletions with continuum configurational bias, ${ }^{29,30}$ which improves the sampling slightly by reducing the number of states which must be visited by the algorithm. We use the resulting chemical potential to calculate the free energy, $A_{\mathrm{ex}} / n k T$, of the system

$$
A_{\mathrm{ex}} / n k T=\mu_{\mathrm{ex}} / k T-Z,
$$

where $Z \equiv P V / n k_{B} T$ is the compressibility factor of the system, and $n$ is the number of copolymer molecules. We can then calculate the entropy, $S / n k$, of the system

$$
S / n k=A / n k T-U / n k T,
$$

where $U$ is the internal energy of the system.

\section{PHASE DIAGRAMS}

Using the simulation technique described in Sec. II, we first focused on the order-disorder transition for symmetric block copolymers. We located $(\chi N)_{\text {ODT }}$ by finding the temperature at which a lamellar system undergoes a phase transition to a disordered state. Figure 2 is a graph of $(\chi N)_{\mathrm{ODT}}$ versus $\bar{N}$ which compares our simulation results for $N=10$ and 20 , and $\eta=0.35,0.40$, and 0.45 to various theoretical results. Our simulation results for $(\chi N)_{\mathrm{ODT}}$ for the 10-bead chains are lower than the predictions of Fredrickson and Helfand. Instead of showing a monotonic trend with $\eta$, the $(\chi N)_{\text {ODT }}$ values are all the same within their error bars. Our simulation results for $(\chi N)_{\text {ODT }}$ for the 20-bead chains are slightly higher than the predictions of Fredrickson and Helfand, and are similar in magnitude to our own results for shorter chains. They do, however seem to decrease slightly with increasing $\eta$ and $\bar{N}$. We do not know the reason why the $(\chi N)_{\text {ODT }}$ values for the 10- and 20-bead chains are essentially the same since the theories of Fredrickson and Helfand and of Barrat and Fredrickson both predict that 




FIG. 2. $(\chi N)_{\mathrm{ODT}}$ for symmetric copolymers as a function of $\bar{N}$. Solid lines are the theoretical predictions by Liebler (Ref. 2), Matsen and Bates (Ref. 7), Fredrickson and Helfand (Ref. 3), and Barrat and Fredrickson (Ref. 4). Open symbols indicate our simulation results.

$(\chi N)_{\text {ODT }}$ should decrease with increasing chain length. However, since neither theory is appropriate for chains as short as $N=20$, the lack of agreement may not be too surprising. Our results can also be compared to those from lattice Monte Carlo simulations. Besold and co-workers used lattice Monte Carlo to look at the order-disorder transition for symmetric chains of length 30 and found $(\chi N)_{\mathrm{ODT}} \approx 23^{14}$, which is only slightly lower than our values of $(\chi N)_{\text {ODT }}$ for chains of length 10 and 20. Larson also used lattice Monte Carlo to examine the order-disorder transition, but included selective solvents in his system that suppressed the disordered phase. Accordingly, his values of $(\chi N)_{\mathrm{ODT}}$ are consistently lower than those we see in our simulations.

By expanding our simulation parameter space to include asymmetric copolymers, we were able to construct phase diagrams showing disordered, lamellar, perforated lamellar, cylindrical and bec spherical structures. Figure 3 display the resulting phase diagrams, plotted in $\chi N$ versus $f$ space for packing fractions $\eta=0.35,0.40$, and 0.45 and chain lengths $N=10$ and $N=20$. The phase diagrams show that a direct phase transition occurs between a disordered phase and a lamellar phase at $f=0.5$. For moderately asymmetric copolymers $(f=0.3,0.4)$, a perforated lamellar (PL) phase is stable below the lamellar phase. For more asymmetric copolymers $(f \leqslant 0.3)$, cylinders are stable between the lamellar phases and disorder (except at $N=10, f=0.3$, and $\eta=0.35$ and 0.40$)$. For highly asymmetric copolymers $(f=0.1), N$ $=20$, and $\eta=0.40$ and 0.45 , bcc spheres form between the cylindrical and disordered phases. We found that the orderdisorder and lamellae-PL transitions are reversible, while the other order-order transitions (cylinders-bcc, PLcylinders, lamellae-cylinders) are only observed during heating.

It is of interest to compare our results to other simulation results and to theoretical predictions. The phase diagrams are consistent with Groot and Madden's simulation study of 10bead chains using dissipative particle dynamics. ${ }^{18}$ They found lamellar, perforated lamellar, cylindrical, and bcc spherical phases in regions of $\chi N$ versus $f$ space similar to those observed by us, although their study did not cover as many values of $\chi N, f$, or $\eta$ as ours. The phase diagrams are generally in agreement with Matsen and Bates' SCFT results, ${ }^{7}$ except that our $(\chi N)_{\text {ODT }}$ values are shifted to higher values of $\chi N$. The phase boundaries above our $(\chi N)_{\text {ODT }}$ are qualitatively similar to those predicted by SCFT, but the values for $(\chi N)_{\text {ODT }}$ are higher than theoretical predictions. Another difference between our phase diagrams and theoretical predictions is that we observe the perforated lamellar (PL) phase between the cylindrical and lamellar phases at $f$ $\geqslant 0.3$, whereas SCFT theory predicts the PL phase to be unstable in this region. ${ }^{7}$ Contrary to those predictions, some experimental studies of copolymers have found the PL phase to be stable (and thermoreversible) in this region. ${ }^{31,32}$ These experimental results are more consistent with our simulation results. Another experimental study found the PL phase to be a metastable phase. ${ }^{33}$

There are a number of possible explanations for the lack of a gyroid phase in our simulations. The gyroid phase is predicted to be stable in the weak segregation regime, but because the ODT is shifted to higher values of $\chi N$, we don't really have a weak segregation regime. While the gyroid phase is also predicted to be stable in the intermediate segregation regime (ISR), we were limited to discrete values of $f$ (the number of $\mathrm{A}$ beads must be an integer) where the gyroid phase is not predicted to be stable. It is also possible that the gyroid phase does not form in our simulations because our box length search algorithm fails to find the right box lengths, causing the PL phase to form instead. To test this, we ran our simulations with 3200 chains for long times near regions of predicted gyroid stability. These systems should have been large enough for the gyroid phase to form without feeling the finite size of the box, but no gyroid structures appeared.

We should also emphasize that the perforations in our perforated lamellar phase are not always hexagonally spaced. While mean-field theories have predicted that the hexagonally perforated lamellae (HPL) phase is not stable, these theories have been unable to treat structures with disordered perforations such as those that we see in our simulations. At $f=0.4$, the perforations are small and transient and do not seem to have an effective repulsive interaction between them (two perforations often appear next to each other), so that the perforations are not ordered in any way. The lack of the PL phase in theoretical phase diagrams could be caused by the inability to handle such disordered structures. At volume fractions of $f=0.3$, the perforations are larger, more stable, appear to repel one another and are ordered (the box was often not large enough to allow perfect hexagonal spacing to form).

At strong segregation (high values of $\chi N$ ) and low volume fractions $(f=0.1$ and 0.2 ), our simulations have produced both lamellar (or perforated lamellar) and cylindrical phases, depending on the initial conditions, at the same temperature. Using the expanded ensemble technique, EVALENCH, ${ }^{19}$ to compare the free energies of the various phases, we found that the lamellar and PL phases are metastable compared to the cylindrical phase over a small range 

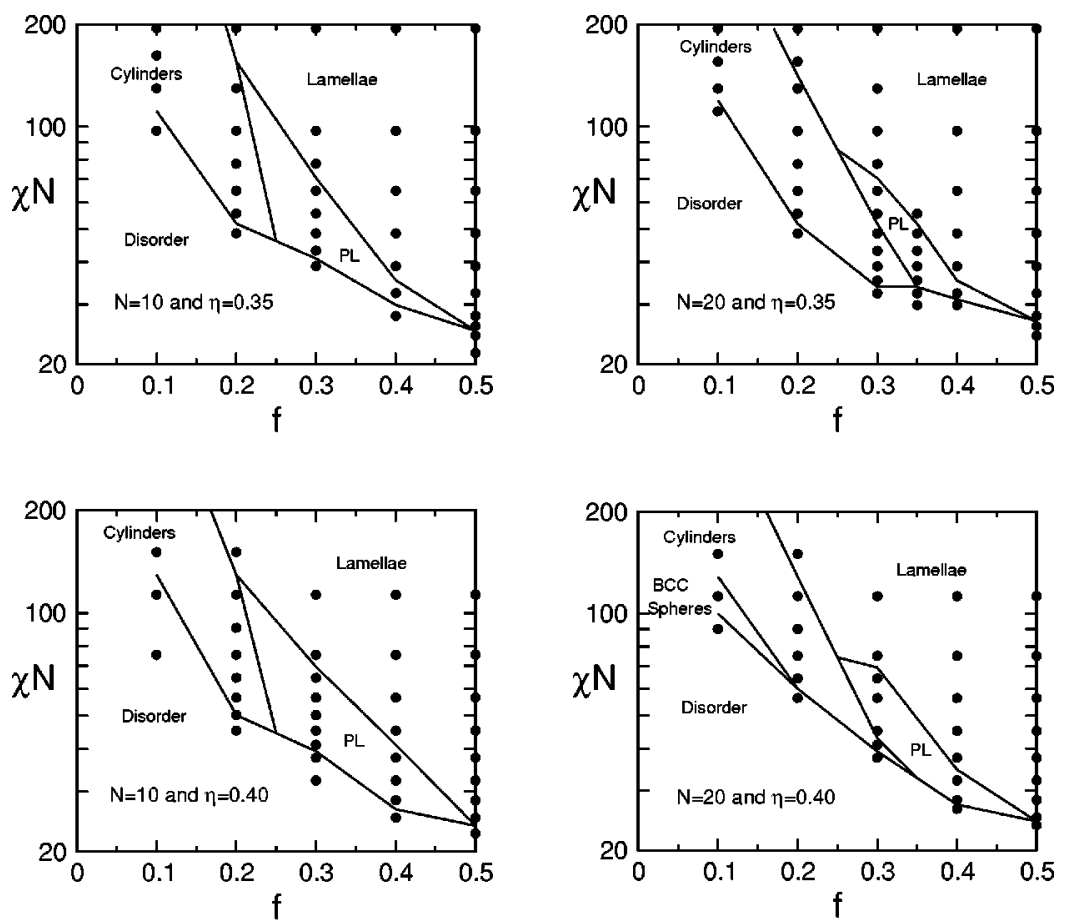

FIG. 3. Simulation phase diagrams for chain lengths 10 and 20 and packing fractions $0.35,0.40$, and 0.45 in $\chi N$ vs $f$ space. Regions of disorder, lamellae, cylinders, perforated lamellae (PL), and bec spheres are shown.


of $\chi N$. For example, the PL phase was present but metastable at $N=10, f=0.1$ and 0.2 , and $\eta=0.35$ and 0.40 . The PL phase was also present at the same conditions and packing fraction 0.45 , but the expanded ensemble technique failed to provide accurate enough values of the free energy to determine the relative stability of the PL and cylindrical phases. In this case we have plotted the PL as being stable in our phase diagram, although the cylinders might be more stable.

Another interesting aspect of our phase diagram is that the metastable cylinders at strong segregation develop an elongated cross section (shown in Fig. 4) similar to those postulated by Burger and co-workers for "super"-strongly segregated copolymers. ${ }^{34}$ We believe our cylinders develop elongated cross sections due to the non-Gaussian stretching of our short chains rather than because of some effect intrinsic to having high values of $\chi N$. While the Gaussian stretching assumption used in most theoretical treatments of copolymers is valid for experimental copolymers $(N \gg 1000)$, the assumption is not as good for short chains. For instance, in Fig. 4, the minority component occupies only two beads along the chain, which means that it cannot stretch much beyond its "Gaussian" conformation. Therefore, in order to increase the size of the cylinders (in order to minimize the interfacial area) as $\chi N$ increases, the cylinders must adopt an elongated cross section. At the same time, the majority block (with 8 beads), is much easier to stretch. This unbalanced stretching leads to an effect similar to conformational asymmetry $\left(a_{\mathrm{A}}<a_{\mathrm{B}}\right)$, which would cause the phase diagram to be shifted to the left. ${ }^{35,36}$ This explains why our phase diagrams are shifted to the left (lower values of $f$ ) in the SSR relative to theoretical predictions.

\section{PROPERTIES OF SYMMETRIC COPOLYMER STRUCTURE}

\section{A. Periodic spacing}

In this section we report the periodic spacing, $d$, of the lamellar structures described in Sec. III. We examine the variation of the periodic spacing with $\chi N$, and compare the results with SCFT and with Barrat and Fredrickson's fluctuation theory. The periodic spacing is calculated by dividing the box length by the number of repeat units in that direction.

Theoretical predictions of periodic spacing behavior can be compared to one another and to simulation results by expressing them in terms similar to Banaszak and Whitmore's prediction ${ }^{11}$ [We have compared our results with Banaszak and Whitmore's results for slightly selective sol- 




FIG. 4. Elongated cylinders at "super-strong" segregation for $\eta=0.40, f$ $=0.2$, and $\chi N=226$. Only the minority component is shown.

vent (their "model System") rather than with Whitmore and Noolandi's 8 results for neutral solvent because Banaszak and Whitmore ${ }^{11}$ reported that the selective solvent results were more accurate and that no differences between selective and neutral solvent spacing behavior were observed using the more accurate algorithm.],

$$
d / a \propto \chi^{p} N^{q} \phi_{c}^{r},
$$

where $\phi_{c}$ is the volume fraction of neutral solvent. Many theories predict that the scaling powers, $p$ and $q$ should be constant. In contrast, SCFT predicts that $p$ and $q$ depend on $\chi N$, solvent volume fraction, and the type of structure. By plotting the spacing versus interaction strength on a $\log -\log$ graph at constant $\eta$ and $N$, we can determine the effective values of the exponent $p$ from our simulations.

Because we have conducted simulations at different packing fractions, we can also examine the effect of packing fraction on spacing. We do this in Fig. 5 by plotting $d / a N^{0.5}$ versus $\chi N$ for a lamellar structure at $f=0.5, \eta=0.35,0.40$ and 0.45 and chain lengths $N=10$ and 20 . We have also included results from Matsen's SCFT and Barrat and Fredrickson's fluctuation theory for comparison. ${ }^{7,4}$ The periodic spacing from our simulations is consistently higher than the SCFT predictions, although agreement does improve with chain length. The simulation values of $p$ determined from Fig. 5 are tabulated in Table II, and indicate that, at least for the short chain lengths studied here, $p$ increases slightly with $N$. This increase in $p$ can be attributed to the non-Gaussian stretching penalties, which are greater for shorter chains. The magnitude of our $p$ values is consistent with the SSR predictions of Banaszak and Whitmore, who found that $p$ varied from 0.2 in the SSR to 0.5 in the WSR. At low values of $\chi N$, Barrat and Fredrickson's predicted spacing is very close to the SCFT results, but deviate from SCFT results as $\chi N$ increases, resulting in a higher value of $p$, which is inconsistent with our own results for short chains.

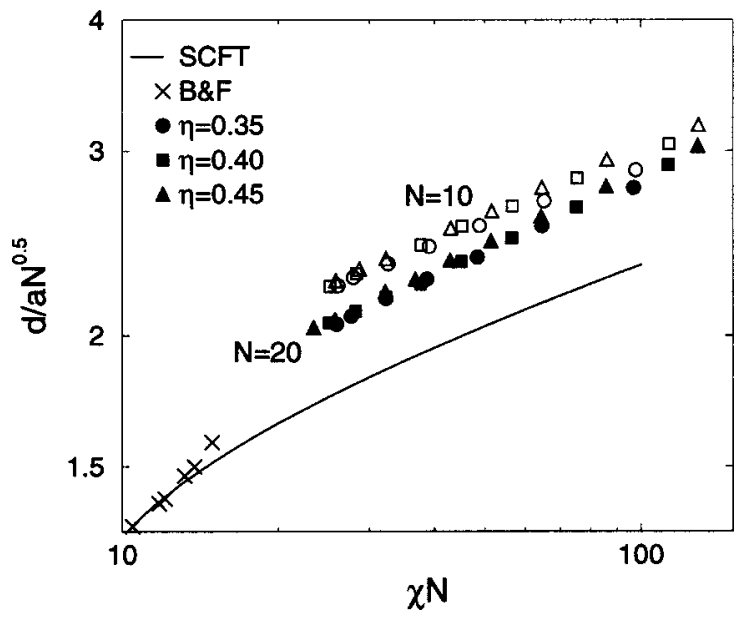

FIG. 5. Graph of $d / a N^{0.5}$ vs $\chi N$ at $f=0.5$ for different packing fractions and chain lengths. Open symbols correspond to $N=10$ systems and closed symbols correspond to $N=20$ systems. Results from Matsen and Bates' selfconsistent theory (Ref. 37) and Barrat and Fredrickson's fluctuation theory (Ref. 4) are also displayed.

Figure 5 suggests that the spacing near the ODT (low values of $\chi N$ ) is unaffected by differences in packing fraction $(r \approx 0)$, although the scaling (with $\chi$ ) of the spacing increases slightly with increasing packing fraction at high values of $\chi N$, resulting in different spacings $(r>0)$. These results seem reasonable when we recognize that a positive value for $r$ indicates a failure of the dilution approximation. The dilution approximation says that solvent particles (or vacancies) distribute themselves evenly throughout the structure, serving only to screen the unfavorable interactions at the interface. In our system, the voids between particles act like a neutral "solvent;" they occupy volume, but do not interact with either component. At low values of $\chi N$, there is not much segregation of the solvent at any packing fraction, so the dilution approximation should be reasonable. However, as $\chi$ increases the solvent is pulled to the interface in order to screen the interactions at the interface more effectively. This is much easier to accomplish at lower densities than at higher densities, so that the polymers do not have to stretch as much to lower the internal energy. This results in different densities having different spacings at high values of $\chi N$ and hence a positive $r$. Contrary to our simulation results, Whitmore and Banaszak reported no change in the periodic spacing with solvent concentration, at constant $\chi_{\mathrm{AB}} \varphi N$ [which is equivalent to our $\chi N$ as indicated in Eq. (7)]. However, because they varied $\chi_{\mathrm{AB}}$ separately from $\varphi$ in coming to this conclusion, any changes due solely to concentration would be difficult to distinguish from changes due to our $\chi$.

Although Murat and co-workers examined the structural spacing of the lamellar structures in their simulations of sym-

TABLE II. Values of $p$ for lamellar structures at different values of $N$ and $\eta$.

\begin{tabular}{ccccccr}
\hline \hline$N$ & & 10 & & 20 & \\
\hline$\eta$ & 0.35 & 0.40 & 0.45 & 0.35 & 0.40 & 0.45 \\
$p$ & 0.19 & 0.21 & 0.21 & 0.21 & 0.22 & 0.23 \\
\hline \hline
\end{tabular}




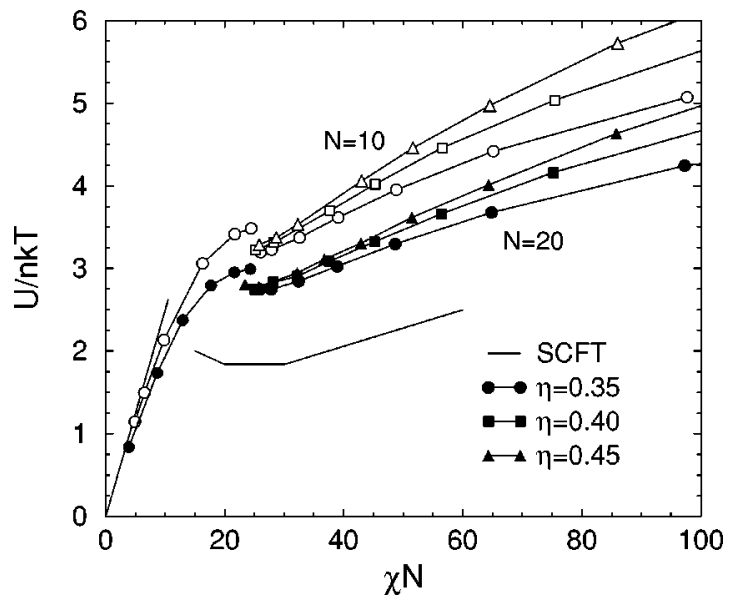

FIG. 6. Graph of $U / n k T$ vs $\chi N$ at $f=0.5$ for different packing fractions and chain lengths. Symbols are the same as those plotted in Fig. 5. Results from Matsen and Bates' SCFT (Ref. 7) are also displayed.

metric copolymers, they did not examine the variation of the structural spacing with interaction strength. ${ }^{17}$ However, by reanalyzing their data (plotting $\ln d$ versus $\ln \epsilon$ ), we find that $p \approx 0.12$ for their data on chains containing 40 to 200 beads. The reason for their low values is unclear.

\section{B. Internal energy of symmetric copolymer structures}

In this section we report values for the internal energy of symmetric copolymer structures obtained from our simulations. We have determined the internal energy directly from the simulation.

We plot the internal energy, $U / n k T$ versus $\chi N$ for symmetric copolymers at $N=10$ and 20 and $\eta=0.35,0.40$, and 0.45 in Fig. 6. The order-disorder transition is indicated by a discontinuity in the data at $\chi N \approx 23$; the disordered phase lies to the left of the discontinuity and the lamellar phase lies to the right. Simulations of disordered structures were only performed for the low packing fraction $(\eta=0.35)$ systems. For disordered structures near the ODT, long simulation times and large simulation boxes were necessary (twice the normal size) because the concentration fluctuations have a long correlation length. However, we expect that the results would be independent of packing fraction since the dilution approximation is valid at these low levels of segregation. We have also plotted Matsen's SCFT results for comparison.

The internal energy per chain of the ordered lamellae is consistently higher than that predicted by theory, but has a similar slope. The 20-bead chains have a lower internal energy per chain than the 10-bead chains, in closer agreement with theory. Preliminary data indicate that concentration fluctuations are responsible for the large difference between theory and simulation. More details on these fluctuations will be included in a later paper.

The effect of packing fraction on internal energy at low segregations is small, consistent with the dilution approximation. However, as the segregation increases, the internal energy is higher for higher packing fractions. This is consistent with solvent segregation, which screens the interactions at

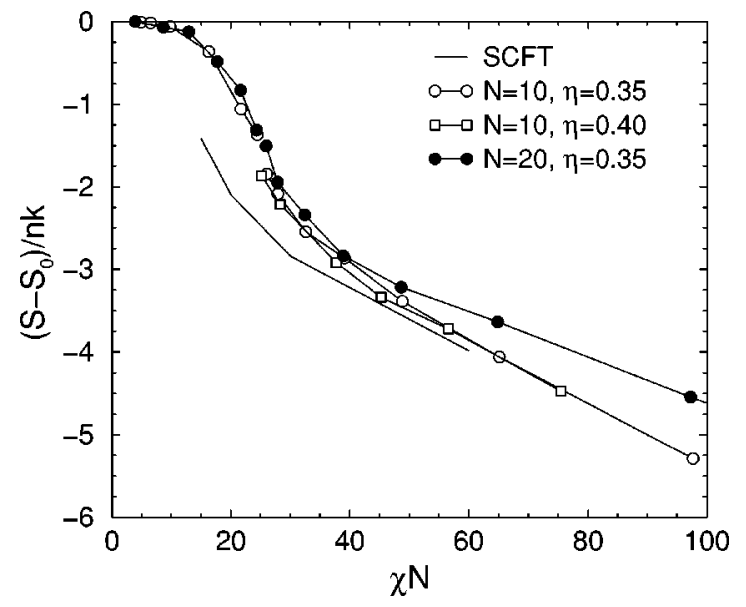

FIG. 7. Graph of $S / n k$ vs $\chi N$ at $f=0.5$ for different packing fractions and chain lengths. Symbols are the same as those plotted in Fig. 5. Results from Matsen and Bates' SCFT (Ref. 7) are also displayed.

the interface more effectively when the packing fraction is low.

The simulation results for disordered structures are more consistent with theoretical predictions than for ordered structures. The data show near-quantitative agreement with mean field theory at very low segregations $(\chi N<5)$. At higher segregations $\left[5<\chi N<(\chi N)_{\mathrm{ODT}}\right]$, the internal energy decreases relative to the mean-field prediction, and continues deviating until the transition occurs. This decrease is consistent with fluctuation theories, which predict that concentration fluctuations form in the disordered morphology at segregations near the ODT. These fluctuations lower the free energy of the disordered state by decreasing the internal energy below the mean-field prediction. Our result that the internal energy of the disordered state for 20-bead chains is lower than that for 10-bead chains contradicts fluctuation theories, which predict that the concentration fluctuations are suppressed as the chain length increases.

\section{Entropy of symmetric copolymer structures}

In this section we report values for the entropy of symmetric copolymer structures obtained from our simulations. We have determined the entropy using the expanded ensemble method described in Sec. II E.

We plot the entropy relative to the homogeneous disordered state, $\left(S-S_{0}\right) / n k$ versus $\chi N$ in Fig. 7 for symmetric copolymers at $N=10$ and $\eta=0.35$ and 0.40 , and at $N=20$ and $\eta=0.35$. The error bars on our entropy values are approximately the same size as the symbols. The orderdisorder transition is indicated by a discontinuity in the data at $\chi N \approx 23$; the disordered phase lies to the left of the discontinuity and the lamellar phase lies to the right.

The entropy of the ordered lamellae is slightly higher than SCFT predictions. The entropy for the two packing fractions at $N=10$ are indistinguishable within their error bars for all values of $\chi N$ we examined. This suggests that although the periodic spacing decreases with solvent segregation, the effect on the entropy is much less significant than the effect on the internal energy (see Fig. 6). The entropy for 
$N=20$ is indistinguishable from the $N=10$ entropy for weak and moderate segregations $\left(\chi_{N}<40\right)$. In this region, the agreement with SCFT does not improve with increasing chain length. At strong segregations $(\chi N>50)$, the entropy for $N=20$ deviates from the entropy of the 10-bead chains because these shorter chains begin to experience nonGaussian stretching. This can be verified by comparing Figs. 5 and 7. The spacing for $N=20$ increases more rapidly than for $N=10$, while the entropy increases more slowly.

\section{SUMMARY}

We have performed discontinuous molecular dynamics simulations of copolymers of varying chain length and volume fraction at different values of $\chi$ and different packing fractions. We have used these simulations to locate $(\chi N)_{\text {ODT }}$ for symmetric copolymers. Our values of $(\chi N)_{\mathrm{ODT}}$ are of the order of magnitude predicted by theory, but do not agree quantitatively with it. The phase diagrams in $\chi N$ versus $f$ space are the most complete block copolymer simulation phase diagrams to date, are in agreement with other simulation results, and are qualitatively consistent with theoretical predictions. While the weak segregation regime is not accessible, the order-order transitions are consistent with theory. We do not see a gyroid phase, but instead see perforated lamellar structures near regions of predicted gyroid stability. Differences between theoretical predictions and our simulation results can be explained in part by the chain lengths considered here, which are much shorter than any considered by theories. Our short chains not only experience fluctuation effects at weak segregation but also experience non-Gaussian stretching effects at strong segregation. Our results for longer chains show better agreement with theory.

We have also examined the properties of the symmetric copolymer systems, including the periodic spacing, internal energy and entropy. We found that the spacing scales as $d$ $\propto \chi^{0.2}$ with slightly higher values of exponents for longer chain lengths and higher packing fractions. These results are in agreement with the SCFT results of Banaszak and Whitmore in the SSR. The magnitude of the spacing is higher than that predicted by theory, but agreement with theory improves for the longer chains. Similarly, the internal energy of the lamellae is higher than that predicted by theory, but agreement with theory also improves with chain length. The internal energy at strong segregation increases with packing fraction due to solvent segregation. The internal energy of the disordered lamellae is lower than that predicted by SCFT and decreases with chain length. The entropy of the symmetric copolymers is higher than SCFT predictions and independent of chain length and packing fraction except at high segregations where the shorter chains experience non-Gaussian stretching.

We hope that the results presented in this paper aid in the development of theories of block copolymer phase behavior that are better able to account for the effects of finite chain length.

\section{ACKNOWLEDGMENTS}

This work was supported by the GAANN Computational Sciences Fellowship of the U. S. Department of Education and the Office of Energy Research, Basic Sciences, Chemical Science Division of the U. S. Department of Energy under Contract No. DE-FG05-91ER14181. Acknowledgment is made to the Donors of the Petroleum Research Fund administered by the American Chemical Society for partial support of this work.

${ }^{1}$ M. W. Matsen, J. Phys.: Condens. Matter 14, R21 (2002).

${ }^{2}$ L. Leibler, Macromolecules 13, 1602 (1980).

${ }^{3}$ G. H. Fredrickson and E. Helfand, J. Chem. Phys. 87, 697 (1987).

${ }^{4}$ J. Barrat and G. H. Fredrickson, J. Chem. Phys. 95, 1281 (1991).

${ }^{5}$ K. R. Schull, Macromolecules 25, 2122 (1992).

${ }^{6}$ E. F. David and K. S. Schweizer, J. Chem. Phys. 100, 7784 (1994).

${ }^{7}$ M. W. Matsen and F. S. Bates, J. Chem. Phys. 106, 2436 (1997).

${ }^{8}$ M. D. Whitmore and J. Noolandi, J. Chem. Phys. 93, 2946 (1990).

${ }^{9}$ A. N. Semenov, Sov. Phys. JETP 61, 733 (1985).

${ }^{10}$ T. Ohta and K. Kawasaki, Macromolecules 19, 2621 (1986).

${ }^{11}$ M. Banaszak and M. D. Whitmore, Macromolecules 25, 3406 (1992).

${ }^{12}$ M. W. Matsen and M. Schick, Phys. Rev. Lett. 72, 2660 (1994).

${ }^{13}$ R. G. Larson, Macromolecules 27, 4198 (1994).

${ }^{14}$ G. Besold, O. Hassager, and O. G. Mouritsen, Comput. Phys. Commun. 122, 542 (1999).

${ }^{15}$ A. Hoffmann, J. Sommer, and A. Blumen, J. Chem. Phys. 106, 6709 (1997).

${ }^{16}$ A. Hoffmann, J. Sommer, and A. Blumen, J. Chem. Phys. 107, 7559 (1997).

${ }^{17}$ M. Murat, G. S. Grest, and K. Kremer, Macromolecules 32, 595 (1999).

${ }^{18}$ R. D. Groot and T. J. Madden, J. Chem. Phys. 108, 8713 (1998).

${ }^{19}$ F. A. Escobedo and J. J. de Pablo, J. Chem. Phys. 103, 2703 (1995).

${ }^{20}$ D. C. Rapaport, J. Chem. Phys. 71, 3299 (1979).

${ }^{21}$ A. Bellemans, J. Orban, and D. V. Belle, Mol. Phys. 39, 781 (1980).

${ }^{22}$ S. W. Smith, B. D. Freeman, and C. K. Hall, J. Comput. Phys. 134, 16 (1997).

${ }^{23}$ M. R. Wilson and M. P. Allen, Mol. Phys. 80, 277 (1993).

${ }^{24}$ N. R. Kenkare, C. K. Hall, and S. A. Khan, J. Chem. Phys. 113, 404 (404).

${ }^{25}$ M. Parrinello and A. Rahman, Phys. Rev. Lett. 45, 1196 (1980).

${ }^{26}$ S. K. Ma, Modern Theory of Critical Phenomena (Benjamin-Cummings, Reading, MA, 1976).

${ }^{27}$ Q. Wang, P. F. Nealey, and J. J. de Pablo, Macromolecules 34, 3458 (2001).

${ }^{28}$ B. Widom, J. Chem. Phys. 39, 2802 (1963).

${ }^{29}$ F. A. Escobedo and J. J. de Pablo, J. Chem. Phys. 105, 4391 (1996).

${ }^{30}$ D. Frenkel and B. Smit, Understanding Molecular Simulation (Academic, San Diego, 1996).

${ }^{31}$ K. Almdal, K. A Koppi, F. S. Bates, and K. Mortensen, Macromolecules 25, 1743 (1992).

${ }^{32}$ A. K. Khandpur, S. Förster, F. S. Bates, and I. W. Hamley, Macromolecules 28, 8796 (1995).

${ }^{33}$ D. A. Hajduk, H. Takenouchi, M. A. Hillmyer, and F. S. Bates, Macromolecules 30, 3788 (1997).

${ }^{34}$ C. Burger, M. A. Micha, S. Oestrech, S. Forster, and M. Antonietti, Europhys. Lett. 42, 425 (1998).

${ }^{35}$ J. D. Vavasour and M. D. Whitmore, Macromolecules 26, 7070 (1993).

${ }^{36}$ M. W. Matsen and F. S. Bates, J. Polym. Sci., Part B: Polym. Phys. 35, 945 (1997).

${ }^{37}$ M. W. Matsen (private communication). 